\title{
Approximate Analytical Model for the Squeeze-Film Lubrication of the Human Ankle Joint with Synovial Fluid Filtrated by Articular Cartilage
}

\author{
Alessandro Ruggiero • Emilio Gòmez • \\ Roberto D'Amato
}

\begin{abstract}
The aim of this article is to propose an analytical approximate squeeze-film lubrication model of the human ankle joint for a quick assessment of the synovial pressure field and the load carrying due to the squeeze motion. The model starts from the theory of boosted lubrication for the human articular joints lubrication (Walker et al., Rheum Dis 27:512-520, 1968; Maroudas, Lubrication and wear in joints. Sector, London, 1969) and takes into account the fluid transport across the articular cartilage using Darcy's equation to depict the synovial fluid motion through a porous cartilage matrix. The human ankle joint is assumed to be cylindrical enabling motion in the sagittal plane only. The proposed model is based on a modified Reynolds equation; its integration allows to obtain a quick assessment on the synovial pressure field showing a good agreement with those obtained numerically (Hlavacek, J Biomech 33:1415-1422, 2000). The analytical integration allows the closed form description of the synovial fluid film force and the calculation of the unsteady gap thickness.
\end{abstract}

Keywords Biotribology - Squeeze-film lubrication . Synovial pressure field - Fluid film force - Analytical model

\footnotetext{
A. Ruggiero $(\bowtie) \cdot$ R. D'Amato

Department of Mechanical Engineering, University of Salerno,

Via Ponte don Melillo, 84084 Fisciano, Italy

e-mail: ruggiero@unisa.it

E. Gòmez

Departamento de Mecánica Industrial, Universidad Politécnica de Madrid, Madrid, Spain
}

\section{Introduction}

Recently there is increasing recognition of the importance of the tribological mechanisms in biomechanics, and in particular, practical interest has been directed to the modelling of human synovial joints lubrication, such as ankle hip and knee joints. It is well known that synovial joints act as mechanical bearings (biobearings) [1] that facilitate the work of the musculoskeletal machine. Depending on the joint location, they may be spherical bearings — such as hip joints, or quasi-spherical bearings—-such as shoulder joints, or the quasi-cylindrical bearings—such as knee, elbow, or ankle joints.

Synovial joints are structured from a pin which is a bone head, and a sleeve constituted by the acetabulum. The pins and sleeves are covered with cartilage that constitutes the structure with antifriction properties. The cartilage structure on the pin surface is more compact than the one on the sleeve surface and should be modelled in most cases as a porous layer. Articular cartilage is a layered medium composed of several structural zones [2]: superficial, middle and deep. The superficial zone of the normal cartilage is the most distinct and reaches up to $200 \mu \mathrm{m}$. Its upper most layer, only about $3 \mu \mathrm{m}$ thick, is called the lamina splendens, is made of non-banded, randomly arranged fine collagen filaments of $4-12 \mathrm{~nm}$ in diameter. Below this layer there are sheets of a wellidentified period, each sheet with predominantly parallel, banded collagen fibrils, their diameter increasing with depth.

Articular cartilage is elastic, fluid-filled and backed by a relatively impervious layer of calcified cartilage and bone. This means that load-induced compression of cartilage will force interstitial fluid to flow laterally within the tissue and to surface through adjacent cartilage. As that area, in turn, 
becomes load bearing, it is partially protected by the newly expressed fluid above it.

The biobearings are lubricated by a synovial fluid. Normal synovial fluid is clear or yellowish; its functions are nutrition and lubrication of cartilage, load bearing and shock absorption.

Recently, the research on synovial joints has focussed on two aspects: to investigate on the fundamental lubrication processes occurring in natural joints, and the development of artificial, replacement joints. Mathematical models of human joints serve to predict quantities which are difficult to measure experimentally, and to simulate changes to the physiological conditions.

With particular reference to the human ankle joint, many researchers have been involved in the modelling of the mechanics of joint lubrication, proposing sophisticated models that involve complex numerical algorithms in order to obtained the desired solution [3-5].

Many theories are based on the hypothesis of boosted lubrication [6]. This lubricating mechanism supposes that the solvent component of SF (considered as a mixture of two incompressible fluids, a viscous-macromolecular complex of hyaluronic acid-protein and the other idealand small-solute-water) flows into the pores of the articular cartilaginous matrix that flattens during the squeeze-action film, so that the concentration of the complex macromolecular acid-hyaluronic protein present in the SF increases until it reaches a limit value. At this point the film fluid is rapidly depleted, and over the majority of contacts between the surfaces, a film of fixed synovial gel forms, preventing the contact and acting as a boundary lubricant during the sliding motion between the two articular surfaces prior to the arrival of the new SF. Naturally during walking, the presence of a fluid film for lubrication of the hinge angular, ensured by the cyclical nature of the load [7], enables the complete filtering of the ideal fluid through the cartilage and thus the formation of the gel synovial.

The aim of this article is to propose a simple analytical squeeze-film lubrication model of the human ankle joint in order to obtain a quick assessment of the fluid film force acting in the articulation by using an analytical solution of the fluid dynamic problem. With this purpose the fluid transport across the articular cartilage has been modelled using the Darcy's equation to depict the synovial fluid motion through a porous cartilage matrix.

\section{Methods}

\subsection{Human Ankle Joint Geometry and Assumptions}

According with the hypothesis of the literature [8] the human ankle joint can be considered as cylindrical, where the motion is allowed only in the sagittal plane; the coupling model is assumed by two infinite rigid circular cylinders (subchondral bone) in the internal contact (a cylinder encased in a cylindrical cavity), covered with thin layer (articular cartilage) of uniform thickness; the lower (talar) articular surface is supposed to be stationary whilst the upper (tibial) surface is assumed to have pure squeeze motion $\dot{e}(t)$ with in half a Newtonian SF film [4, $5,7]$. The last assumption was further justified in the article [9] in which has been observed that the effect of increased viscosity of the normal SF for low shear rates does not influence the fluid film thickness for entraining velocities encountered in walking; it follows that the SF in the ankle joint can be assumed as Newtonian during walking.

Figure 1 shows the model and the coordinates $x$, $y$ used. It is assumed that the ankle is loaded in the $y$ direction in the plane of symmetry by a load $W(t)$ $[\mathrm{N} / \mathrm{m}]$, as occurs during walking. The movement occurs in the $x-y$ plane. Articular surfaces move along $y$. The surface with its centre at $x=0$ is bounded with the edges at $x= \pm a$ (i.e. $\theta= \pm \frac{a}{R}= \pm \beta$ with $\frac{1}{R}=\frac{1}{R_{\mathrm{t}}}+\frac{1}{R_{\mathrm{I}}}$ ) in which the synovial pressure is assumed to be equal to zero. Only the symmetrical case is considered in this article allowing the generalisation of the obtained analytical results to the other non-symmetrical configurations. For cartilage the linear mixture model [10] could be adopted, where the linear (small deformation) homogeneous and isotropic elastic porous cartilage matrix is considered with the ideal interstitial fluid, flowing due to the fluid pressure gradient through the porous matrix.

The adopted gap geometry is qualitatively confirmed by some numerical investigation made by the authors on real plain radiograph images and on real computed axial tomography data (TAC) (Fig. 2).

Starting from TAC data, by using first the Hounsfield unit scale, it has been possible to make a threshold of talar

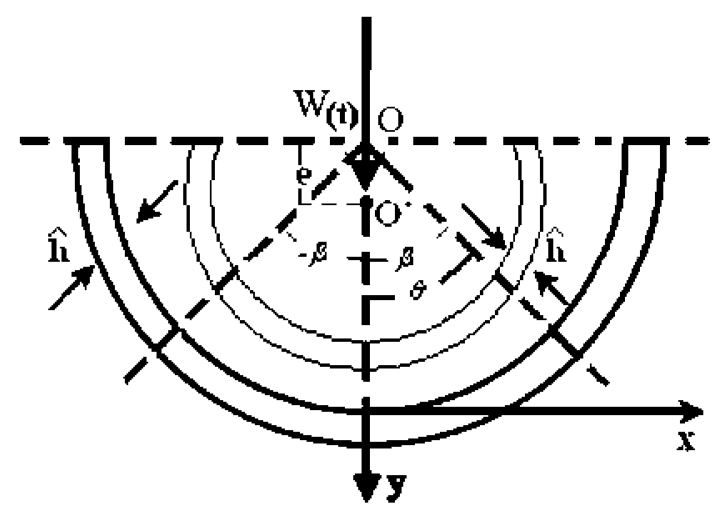

Fig. 1 Human ankle joint geometry 


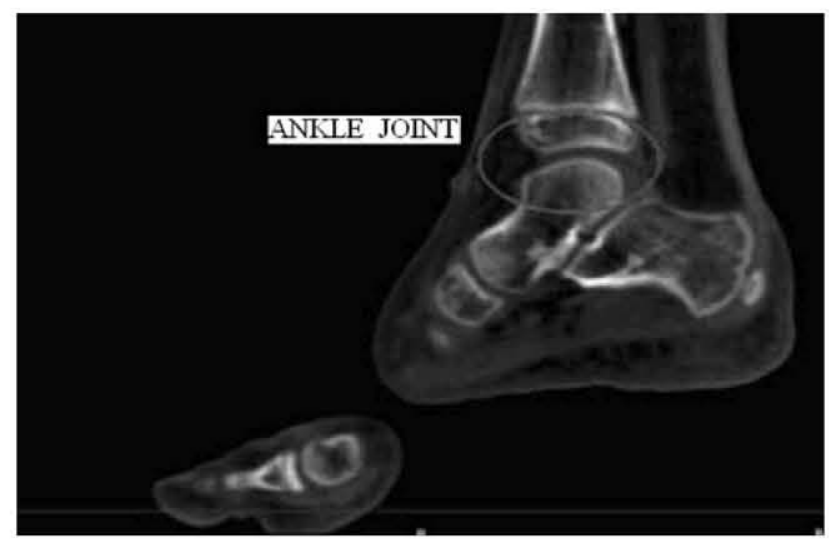

Fig. 2 Radiograph images of the human ankle joint (side view)

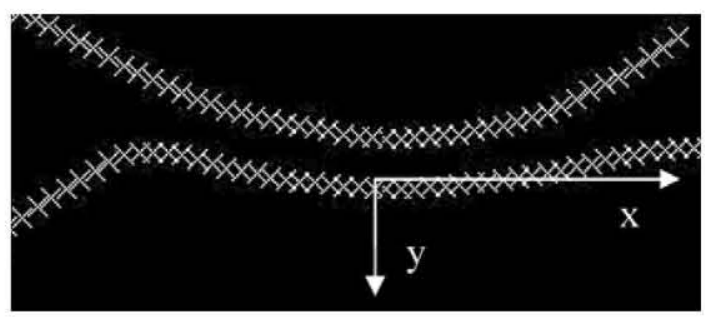

Fig. 3 Plain representation of contact in correspondence of the middle $x-y$ plane

and tibial surface. By interpolating the obtained points it has been possible to reconstruct the shape of the surfaces.

The plain representation of them, in correspondence of the middle $x-y$ plane (Fig. 3), confirms the validity of the chosen geometry.

\subsection{Mathematical Model}

The thickness of the gap of two infinite circular cylinders can be written as [5]:

$h(x, t)=\tilde{h}(t)-2 u(x, t)-2 \hat{h}$

where $\tilde{h}(t)$ is the SF film thickness including cartilage surfaces, $u(x)$ is the displacement in the vertical direction of the articular surface and $\hat{h}$ is the thickness of the cartilage surfaces; $u(x)$ can be evaluated through the approximation due to Mattheson [11] and Armstrong [12]. In order to evaluate the component displacement due to cartilage deformation these authors considered the constitutive equations of the latter, linear elastic material, related to the Cartesian plane $x_{i}(i=1,2,3)$ transversely isotropic with the axis $x_{3}$. If there are no restrictions on the component $\varepsilon_{i j}$, it is possible to get $\sigma_{i j}$ in the form:

$$
\begin{aligned}
{\left[\begin{array}{l}
\sigma_{11} \\
\sigma_{22} \\
\sigma_{33} \\
\sigma_{13} \\
\sigma_{23} \\
\sigma_{12}
\end{array}\right]=} & {\left[\begin{array}{cccccc}
C_{11} & C_{12} & C_{13} & 0 & 0 & 0 \\
C_{21} & C_{22} & C_{23} & 0 & 0 & 0 \\
C_{31} & C_{32} & C_{33} & 0 & 0 & 0 \\
0 & 0 & 0 & 2 \mu_{13} & 0 & 0 \\
0 & 0 & 0 & 0 & 2 \mu_{13} & 0 \\
0 & 0 & 0 & 0 & 0 & 2 \mu_{12}
\end{array}\right] } \\
& *\left[\begin{array}{l}
\varepsilon_{11} \\
\varepsilon_{22} \\
\varepsilon_{33} \\
\varepsilon_{13} \\
\varepsilon_{23} \\
\varepsilon_{12}
\end{array}\right]
\end{aligned}
$$

with

$$
\begin{aligned}
& C_{11}=E_{1}\left(1-v_{31}^{2} E_{1} / E_{3}\right) /\left(1+v_{21}\right) \Delta \\
& C_{12}=E_{1}\left(v_{21}+v_{31}^{2} E_{1} / E_{3}\right) /\left(1+v_{21}\right) \Delta \\
& C_{13}=E_{1} v_{31} / \Delta \\
& C_{33}=E_{3}\left[1+2 v_{31}^{2} E_{1} /\left(E_{3} / \Delta\right)\right] \\
& \Delta=1-v_{21}-2 v_{31}^{2} E_{1} / E_{3}
\end{aligned}
$$

$\sigma_{i j}(i, j=1,2,3)$ is the cartilage components stress tensor, $\varepsilon_{i j}$ components tensor of small deformations. $v_{i j}=$ $-\frac{\varepsilon_{j j}}{\varepsilon_{i i}}(i \neq j)$ and $E_{i}=\frac{\sigma_{i i}}{\varepsilon_{i i}}$ are the Poisson ratios and Young's modulus with strain due to the uniaxial component $\sigma_{i i}$, respectively, whilst $\mu_{13}, \mu_{12}$ represent the shear modulus, with $\mu_{12}=\frac{E_{1}}{2\left(1+v_{21}\right)}$.

In the case of cylindrical geometry, it is possible to evaluate the vertical displacement $u(x)$ of the articular surface as [13]:

$u(x)=\frac{h^{3}}{3 \mu} \frac{\partial^{2} S}{\partial x^{2}}$

In order to obtain an analytical approximate solution for the pressure field, in this article, the effects of cartilage deformations are neglected. In fact as already reported in the literature this condition occurs for light load.

With reference to the scheme in Fig. 1, under the classical hypotheses of hydrodynamic lubrication, the Reynolds equation for the contact in the case of pure squeeze motion can be written as follows:

$\frac{\partial}{\partial x}\left(\frac{h^{3}}{\mu} \frac{\partial p}{\partial x}\right)+\frac{\partial}{\partial z}\left(\frac{h^{3}}{\mu} \frac{\partial p}{\partial z}\right)=12\left(W_{\mathrm{ul}}\right)$

in which the effective velocity component $W_{\mathrm{ul}}$ is composed of two terms: $W \mathrm{p}$ is the $y$-velocity of the talar surface and $W_{0}$ is the fluid-velocity due to the permeability of the cartilage matrix.

$W_{\mathrm{ul}}=W_{\mathrm{p}}-W_{0}$

The $W_{0}$ component can be calculated using Darcy's equation: 
$W_{0}=\frac{\Phi H}{\mu}\left(\frac{\partial^{2} p}{\partial x^{2}}+\frac{\partial^{2} p}{\partial z^{2}}\right)$

in which $\Phi=k \mu\left[\mathrm{m}^{2}\right]$ is the permeability factor of the cartilage porous matrix and $H=2 \hat{h}$

$W_{\mathrm{p}}=\frac{\partial h}{\partial t}$

By substituting the Eq. 6 in the Eq. 4 the modified Reynolds equation can be written as follows

$\frac{\partial}{\partial x}\left[\left(h^{3}+12 \Phi H\right) \frac{\partial p}{\partial x}\right]+\frac{\partial}{\partial z}\left[\left(h^{3}+12 \Phi H\right) \frac{\partial p}{\partial z}\right]=12 \mu \frac{\mathrm{d} h}{\mathrm{~d} t}$

with

$h(x, t)=\tilde{h}(x, t)-2 \hat{h}$

in which $\tilde{h}(t)$ is the fluid film thickness including the cartilage layers $(\hat{h})$.

Assuming mono-dimensional pressure in the $x$ direction, the Eq. 8 becomes:

$\frac{\partial}{\partial x}\left[\left(h^{3}+12 \Phi H\right) \frac{\partial p}{\partial x}\right]=12 \mu \frac{\mathrm{d} h}{\mathrm{~d} t}$

By using polar coordinates:

$\frac{1 \partial}{R^{2} \partial \theta}\left[\left(h^{3}+12 \Phi H\right) \frac{\partial p}{\partial \theta}\right]=12 \mu \frac{\mathrm{d} h}{\mathrm{~d} t}$

with

$\tilde{h}(\theta, t)=c+e \cos \vartheta$

where $c$ and $e$ are the biobearing clearance and eccentricity, respectively.

The pressure field is obtained by integrating (11):

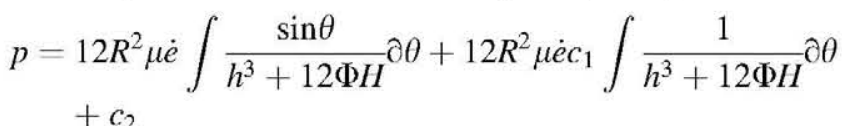

with $c_{1}$ and $c_{2}$ determined by imposing void values of pressure at $\pm \frac{a}{R}= \pm \beta$,

In order to obtain the approximate functions, (8) has been replaced with:

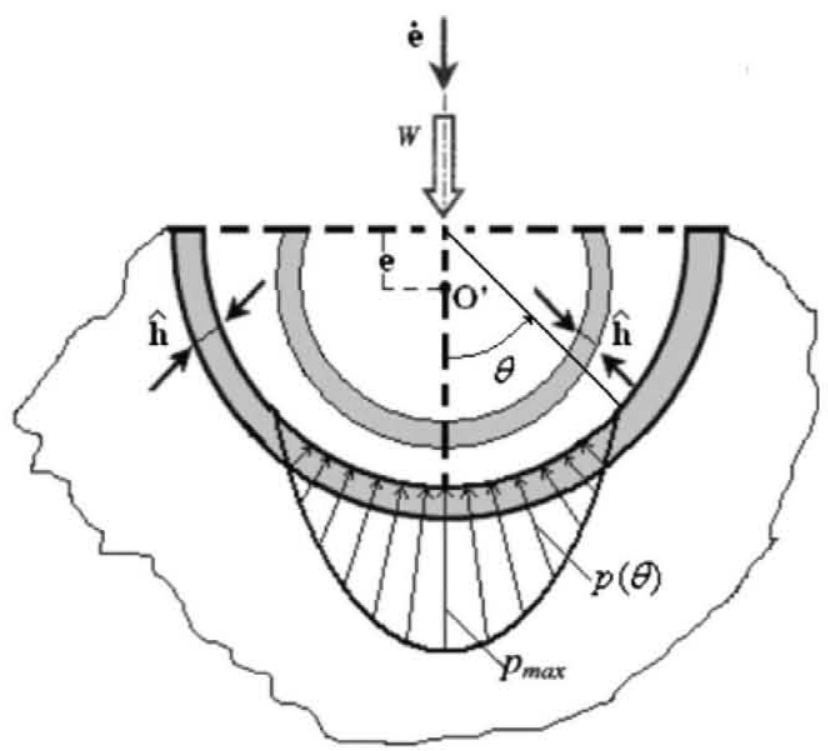

Fig. 4 Schematic view of the fluid film pressure

in which the term $h(\theta)^{3}+12 \Phi H$ has been substituted with the well-fitting function $(h(\theta)+12 \Phi H-6 \Phi e)^{3}$ obtained from similar expressions used in [14] (Fig. 4).

The synovial pressure field can be written by integrating (14) in the closed form as:

$p(\theta)=\frac{12 R^{2} \mu \dot{e}\left[-\frac{1}{c-6(-2+e) \Phi+e \cos \beta^{2}}+\frac{1}{c-6(-2+e) \Phi+e \cos \theta^{2}}\right]}{2 e}$

The analytical form of the hydrodynamic pressure allows to calculate the fluid film force:

$f_{\mathrm{r}}=L \int_{-\beta}^{\beta} p(\theta) \cos (\theta) R \mathrm{~d} \theta$

By solving analytically (16) we obtain:

$f_{\mathrm{r}}=12 R^{3} \mu \dot{e} L\left\{\frac{2(c+12 \Phi) \arctan h\left[\frac{(c-e+12 \Phi) \tan (\beta / 2)}{\sqrt{-c^{2}+e^{2}-24 c \Phi-144 \Phi^{2}}}\right]}{e\left(-c^{2}+e^{2}-24 c \Phi-144 \Phi^{2}\right)^{3 / 2}}-\frac{\frac{\beta}{e}+\frac{(c+12 \Phi+e \cos \beta) \sin \beta}{\left(c^{2}-e^{2}+24 c \Phi+144 \Phi^{2}\right)}}{c+12 \Phi+e \cos \beta^{2}}\right\}$ 


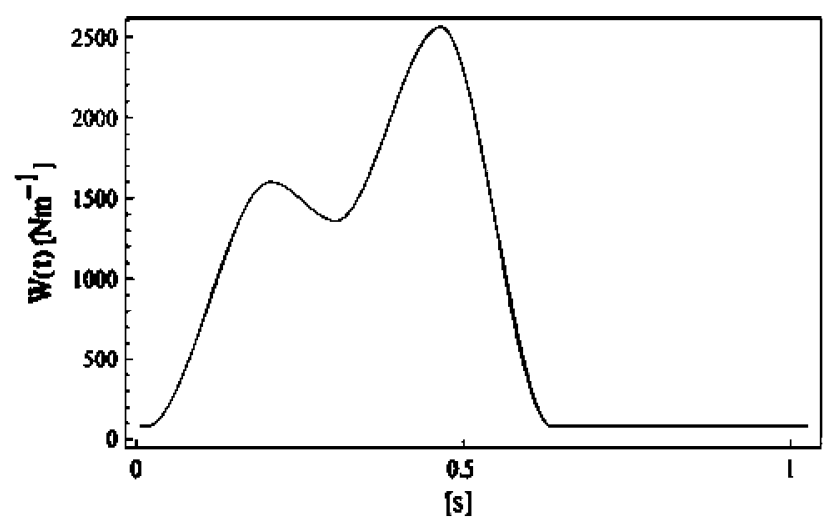

Fig. 5 Variation of $W(t)$ in the ankle joint during walking [5]

By equating the fluid film force (17) to the external load $W(t)$, representing the load cycle during the walking [5] see Fig. 5, and neglecting the others forces acting on the joint, it is possible to write the equilibrium equations for the talar surface in the $x-y$ plane as follows:

$f_{\mathrm{r}}(e, \dot{e})=W(t)$

Equation 18 represents a non-linear differential equation. The solution provides the time history of the functions $e(t)$ and $\dot{e}(t)$.

\section{Results}

The numerical integration of the Eq. 18 has been performed by using a predictor-corrector Adhams method with $\left.e\right|_{t=0}=\bar{h}$, where $\bar{h}$ is the fluid film thickness in absence of the load.

In order to compare the results with those obtained in [15] the same typical numerical values have been adopted for the calculations (see Appendix B).

The calculation of the $e(t)$ function provides the evolution of the velocity $\dot{e}(t)$ (Fig. 6) and of the fluid film thickness $h(0, t)$ (Fig. 7) during the imposed load (walking).

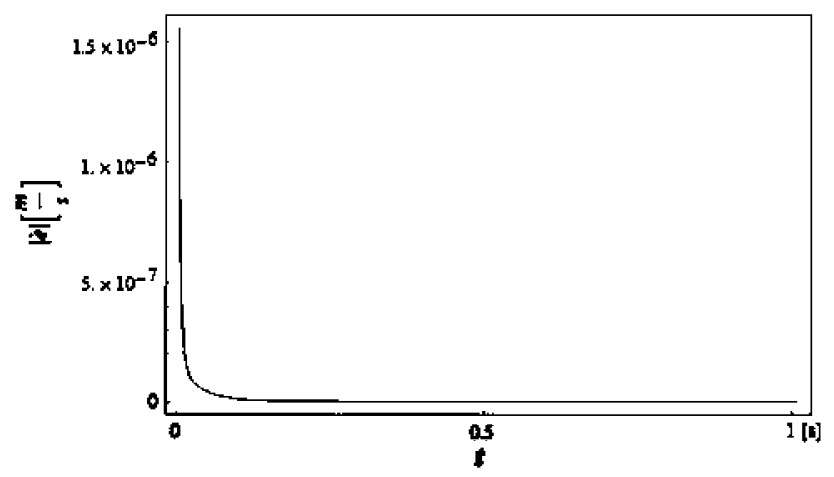

Fig. 6 Variation of $\dot{e}(t)$ in the ankle joint during walking
In Fig. 8 a typical synovial pressure field is shown by varying the angular coordinate $\theta$ and the time during the first $0.05 \mathrm{~s}$ of the walking when using the results obtained from the solution of (18).

In Fig. 9, for different values of $\phi$, the synovial pressure field versus $\theta$ is shown in correspondence of the instant in which the load is applied.

The plot in Fig. 9 shows the synovial pressure field versus $\theta$ at the start of the walking $(t=0+)$; the obtained values have been compared with those reported in [15] and show a good agreement.

\section{Discussion}

This article focusses on the possibility of obtaining an analytical approximate squeeze-film lubrication model of the human ankle joint, for a quick assessment of the synovial pressure field and of the load carrying of the human articulation during walking, due to the squeeze motion of the hinge, with sliding zero. After a numerical investigation on real radiographic data confirms the simplified geometry adopted in this article, a simple model of the human ankle joint is proposed, in order to generate an approximate analytical expressions of the synovial pressure field and of the unsteady fluid film force as function of the eccentricity ratio and squeeze film.

The results indicate that for normal approach motion of the human hinge, a jump in the fluid pressure at the articular surface occurs at the moment of a step-load application (Fig. 8), raising values to about $1.5 \mathrm{MPa}$ at $h\left(0, t=0^{+}\right)$. This jump makes water imbibe into the cartilage, and the SF film formed. Synovial fluid filtration in mainly controlled by the cartilage permeability coefficient $\phi$, which describes the way that water and other low molecular weight substances are infiltrated into the pores of the talar surface cartilage. As the macromolecules of the hyaluronic acid-protein complex are too large to penetrate into the pores, their concentration increases in the synovial film and a gel forms on the surface which prevents dry contact. The gel serves as a boundary lubricant if a sliding motion follows before a fresh $\mathrm{SF}$ gets into the contact.

The results in Fig. 7a show the evolution of $h(0, t)$ for $R=0.35$ and $R \rightarrow \infty$ (parallel surfaces) before the gel forming phase, and confirm the investigation made by Hlavacek in [15] by using a more complicated numerical model [16-18]. Furthermore these results do not exclude the possibility that a continuous fluid film may be maintained in normal human ankle joint during walking, where sliding occurs and a fresh SF comes into contact from outside. With normal articular cartilage, maintenance of a lubricating fluid film is much aided by the cyclic nature of the loading encountered in walking, compared with the 

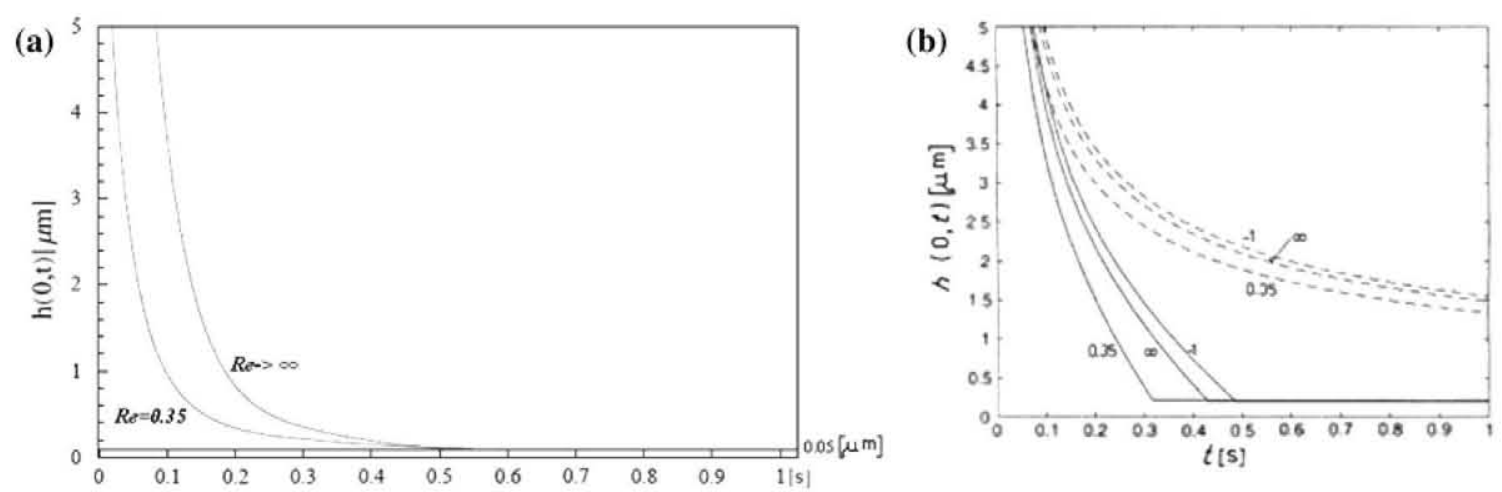

Fig. 7 a Evolution of $h(0, t)$ for $R=0.35$ and $R \rightarrow \infty$, b results obtained by Hlavacek [15]

Fig. 8 Fluid film pressure field by varying the angular coordinate $\theta$ and time

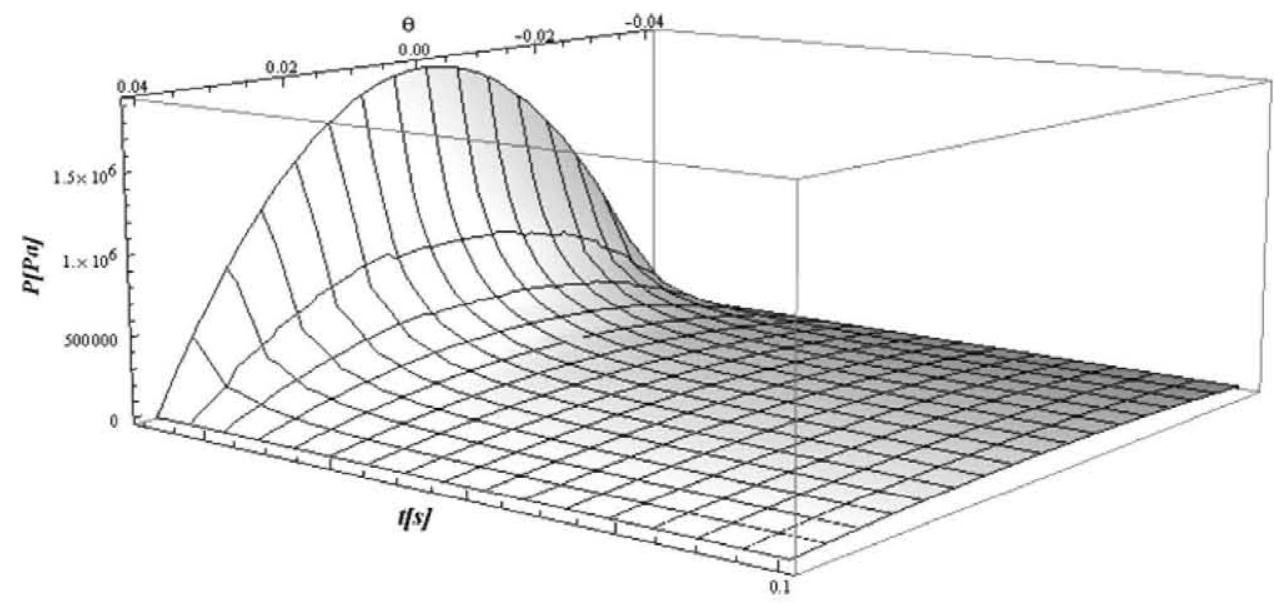

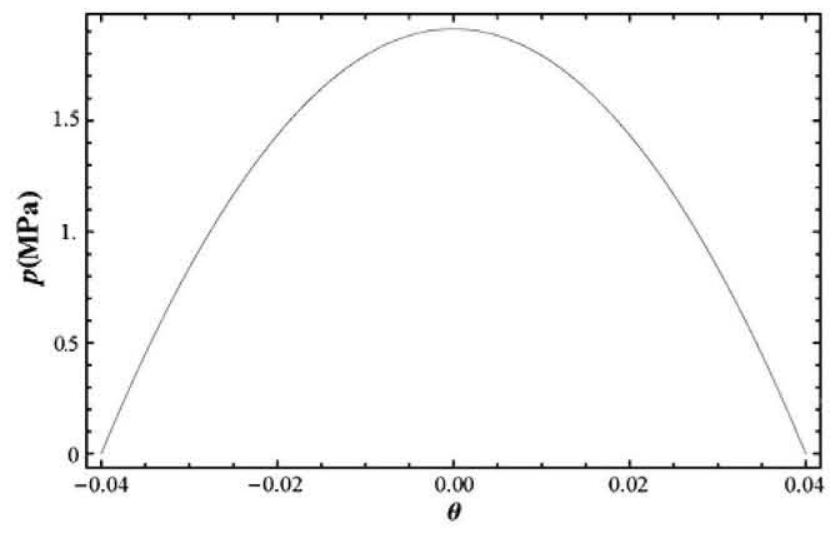

Fig. 9 Variation of synovial pressure at the start of the walking

steady loading in standing, where the fluid film is quickly filtered out into a protective permanent gel film. By contrast, in the pathological case, the protective synovial gel layer is quickly depleted after several steps and the surfaces may come briefly into contact in each cycle.

The results of the current simplified lubrication model underline that:

- due to the adopted hypotheses, the proposed model gives better results in the cases in which it is possible to neglect the effects of the cartilage deformations and of the synovial gel boundary lubricant formation (i.e. low loaded joints).

- the closed form solutions for the synovial pressure field and of the fluid film force are useful in all the dynamic analyses of the ankle joints in which the aim is to obtain a better trade-off between accuracy and computational expense for a quick assessment of the forces acting in the synovial joint during a pure squeeze motion. It gives a better readability of all the principal geometrical and rheological parameters that characterise the synovial joint, allowing to cover not only the individual case, but also the whole class to which the system belongs.

It should be noted in conclusion that this article represents a preliminary analysis of a squeezed synovial film and many simplifying assumptions have been adopted in the mathematical modelling in order to receive an analytic solution. These are, amongst others, hydrodynamic lubrication, squeeze motion, permeability Darcy's model, rigid cartilage matrix and subchondral bone, Newtonian SF. In the literature these assumptions are accepted in some cases, but the Authors hope to obtain in future some experimental verification in order to support the approximate theoretical prediction obtained and/or improve the proposed method, 
first removing the hypothesis of rigid cartilage surfaces, i.e. investigating on the possibility to propose an analytical or semi-analytical micro-elastohydrodynamic model, to taking into account the deformation of these surfaces and its effects on the dynamic behaviour of the hinge.

\section{Appendix A}

\begin{tabular}{ll} 
Nomenclature \\
\hline$a$ & Tibial length \\
AC & Articular cartilage \\
$E$ & Young's modulus of the cartilage matrix \\
$h(x, t)$ & Synovial film thickness \\
$\phi$ & Permeability of the cartilage matrix \\
$c$ & Clearance \\
$p(x, t)$ & Synovial pressure \\
$q(x, t)$ & Synovial flow through the cartilage matrix \\
$f_{\mathrm{r}}$ & Synovial fluid film force \\
$e(t)$ & Squeeze velocity \\
$R$ & $1 / R=1 / R_{1}-1 / R_{\mathrm{u}}$ effective radius curvature of the contact \\
$R_{\mathrm{l}}, R_{\mathrm{u}}$ & Radius curvature of SL and Su \\
$s_{1}, s_{\mathrm{u}}$ & Inferior articular surface (thalamus) and upper (tibial) \\
$\mathrm{SF}$ & Synovial fluid \\
$t$ & Time \\
$u(x, t)$ & Displacement of the articular surface \\
$W(t)$ & Periodic load for unit of length \\
$\mu$ & Shear modulus of the cartilage matrix \\
$\eta$ & Viscosity of synovial fluid
\end{tabular}

\section{Appendix B}

Table numerical values used for the calculations

\begin{tabular}{lll}
\hline Parameters & Numerical values & Units \\
\hline$a$ & $14 \times 10^{-3}$ & {$[\mathrm{~m}]$} \\
$L$ & $28 \times 10^{-3}$ & {$[\mathrm{~m}]$} \\
$\hat{h}$ & & {$[\mathrm{~m}]$} \\
$c$ & $7.25 \times 10^{-7}$ & {$[\mathrm{~m}]$} \\
$R$ & $3.5 \times 10^{-1}$ & {$[\mathrm{~m}]$} \\
$R_{1,}$ & $22 \times 10^{-3}$ & {$[\mathrm{~m}]$} \\
$\phi$ & $2 \times 10^{-14}$ & {$[\mathrm{~m} / \mathrm{s}]$} \\
$\beta$ & $4 \times 10^{-2}$ & $\mathrm{rad}$ \\
$\mu$ & $10^{-2}$ & {$[\mathrm{~Pa} \mathrm{~s}]$} \\
\hline
\end{tabular}

\section{References}

1. Walicki, E., Walika, A.: Mathematical modelling of some biological bearings. Smart Mater. Struct. 9, 280-283 (2000)

2. Lane, J.M., Weiss, C.: Review of articular cartilage collagen research. Arthritis Rheum. 18, 553-562 (1975)

3. Sep, J., Pietal, A.K.: Applications of finite element method in synovial joint numerical calculations. In: Proceedings of the International Multiconference on Computer Science and Information Technology, pp. 449-455. ISSN 18967094 (2007)

4. Hlavacek, M., Vokoun, D.: Elastohydrodynamic lubrication of cylinders with soft incompressible coatings firmly bonded to the substrate. Wear 197, 1-8 (1996)

5. Hlavacek, M.: Squeeze-film lubrication of the human ankle joint subjected to the cyclic loading encountered in walking. J. Tribol. 127, 141-148 (2005)

6. Walker, P.P., Dowson, D., Longfield, M.D., Wright, V.: "Boosted lubrication" in synovial joints by fluid entrapment and enrichment. Ann. Rheum. Dis. 27, 512-520 (1968)

7. Medley, J.B., Dowson, D., Wright, V.: Transient elasto-hydrodynamic lubrication models for the human ankle joint. Eng. Med. 13, 137-151 (1984)

8. Medley, J.B., Dowson, D., Wright, V.: Surface geometry of the human ankle joint. Eng. Med. 12(1), 35-41 (1983)

9. Hlavacek, M.: A central film thickness formula for elastohydrodynamic lubrication of cylinders with soft incompressible coating and non Newtonian piecewise power-law lubricant in steady rolling motion. Wear 205, 20-27 (1996)

10. Mow, V.C., Kuei, S.C., Lai, W.M., Armstrong, C.G.: Biphasic creep and stress relaxation of articular cartilage: theory and experiment. Trans. ASME J. Biomech. Eng. 102, 73-84 (1980)

11. Matthewson, M.J.: Axi-symmetric contact on thin compliant coatings. J. Mech. Phys. Solids 29, 89-113 (1981)

12. Armstrong, C.G.: An analysis of the stress in a thin layer of articular cartilage in a synovial joint. Eng. Med. 15, 55-61 (1986)

13. Hlavacek, M.: Frictionless contact of two parallel congruent rigid cylindrical surfaces coated with thin elastic transversely isotropic incompressible layers: an analytic solution. Eur. J. Mech. A Solids 25, 497-508 (2006)

14. D'Agostino, V., Ruggiero, A., Senatore, A.: Approximate model for unsteady finite porous journal bearings fluid film force calculation. Proc. Inst. Mech. Eng. J J. Eng. Tribol. 220, 227-234 (2006)

15. Hlavacek, M.: Squeeze-film lubrication of the human ankle joint with synovial fluid filtrated by articular cartilage with the superficial zone worn out. J. Biomech. 33, 1415-1422 (2000)

16. Hlavacek, M.: The role of synovial fluid filtration by cartilage in lubrication of synovial joints-I. Mixture model of synovial fluid. J. Biomech. 26(10), 1145-1150 (1993)

17. Hlavacek, M.: The role of synovial fluid filtration by cartilage in lubrication of synovial joints-II. Squeeze-film lubrication: homogeneous filtration. J. Biomech. 26, 1151-1160 (1993)

18. Hlavacek, M.: The role of synovial fluid filtration by cartilage in lubrication of synovial joints-IV. Squeeze-film lubrication: the central film thickness for normal and inflammatory synovial fluids for axial symmetry under high loading conditions. J. Biomech. 28, 1199-1205 (1995)

19. Maroudas, A.: Studies on the formation of hyaluronic acid films. In: Wright, V. (ed.) Lubrication and Wear in Joints, pp. 124-133. Sector, London (1969) 